\title{
Sina Weibo Mood Predicting the Economics Trends
}

\author{
Fan PAN \\ Wuhan Institute of Technology, College of Post and Telecommunication, Wuhan, P. R. China
}

Hui LI

Wuhan Institute of Technology, School of Computer Science and Engineering, Wuhan, P. R. China

\begin{abstract}
Social media has already become an important part of daily life. Entering the era of smart phone, people always share their created contents anywhere anytime. Among the created contents, the microblog has become a mapping of human life, which can clearly express natural human emotion and mood. In this paper, we demonstrate how social media content generated by microblog can be used to predict realworld economics trends. Especially, we used the most popular Chinese microblog services Sina Weibo to provide the collective big data for forecasting China economics trends such as China's Shanghai securities (SSE) composite index. A simple model built based on the social mood at which Weibo are created about people emotion can outperform market-based predictors. We further demonstrate how sentiments extracted from Weibo can be further utilized to improve the predicting power of social media.
\end{abstract}

KEYWORD: Social Computing; Sina Weibo; Mood analysis; Economics trends prediction

\section{INTRODUCTION}

Economic prediction has attracted much attention from academia as well as business proposed by Bollen (2011), Boyacioglu (2010), and Vui (2013). Social media has exploded as a category of online discourse where people create content, share it, express mood and network at a prodigious rate. China internet market is very different from the western internet market by many factors, but some nearly similar western famous internet companies can be homogonously found in China. For example, Twitter is an extremely popular online micro blogging service, launched on July 13 2006. It has a very large user base, consisting of several hundred millions of users from the whole world. For Chinese government policies of internet management, Twitter is not now launched in China. However, Weibo was released by the Sina Corporation, China's biggest web portal, in August 2009. According to the Sina corporation annual report, the Weibo microblog now has more than five hundred million registered users, and one hundred million active users. Because of its ease of use, speed and reach, social media, just like Weibo, is fast changing the public discourse in society and setting trends and agendas in topics that range from the top-news and economics to technology and all aspects of daily social life.

Since social media can also be constructed as a form of collective big data, we can use it to investigate its power at predicting the economic trends. As known, news most certainly influences stock market prices, public mood states or sentiment may play an equally important role proposed by Garcia (2013). And we also know from psychological research that emotions, in addition to information, play a significant role in human decision-making proposed by Van Kleef (2010). Behavioral finance has provided further proof that financial decisions are significantly driven by emotion and mood proposed by Fama (1998). Thus, it is therefore reasonable to assume that the public mood and sentiment can drive economics trends as much as news proposed by Soroka (2006).

In the case of social media, the information that propagates through large user communities presents an interesting opportunity for harnessing that data into a form that allows for specific predictions about particular outcomes, without having to institute market mechanisms. One can also build models to aggregate the opinions of the collective population and gain useful insights into their behavior, while predicting economics trends.

In this paper, we investigate whether public sentiment, as expressed in large-scale collections of daily Weibo posts, can be used to predict the China's Shanghai securities (SSE) composite index. We divide variation in the public mood from Weibo submitted to the Weibo service from November 1, 
2012 to December 1, 2013 (contains the important day of 18th National Congress of the Communist Party of China and the 3rd Plenary Session of 18th Communist Party of China Central Committee). We analyses the text content of Weibo submitted on everyday to provide a positive or negative daily time series of public mood. The resulting public mood time series are correlated to the SSE composite index to access their ability to predict changes in the SSE composite index over time. Our results indicate that the prediction accuracy of standard stock market prediction models is significantly improved when certain mood dimensions are included, but not others.

\section{SOCIAL MOOD AND ECONOMICS}

\subsection{The relationship between social mood and economics}

As the previous illustrates, people's attitudes are not based solely on their independent analysis. Interaction with others has a strong influence and leads to a shared emotion, or social mood. Collectively shared opinions and beliefs shape individual decisions, which aggregate into social trends, fashion, and action. The economy is the sum of the economic interactions in society. One person's decision to buy a house or not, or risk the family's capital on investment or not, may not effect the economy. However, if the social mood influences many people to buy houses and expand their investments, then the economy expands. That is, the level of social mood affects the economy because participants interact. The media, economists, and investors also seem to find the relation between mood and the economy important as evident by the considerable attention given to the Consumer Confidence data released monthly by The China's National Bureau of Statistics. We would go one step further and propose that economic optimism is a subset of social optimism. A substantial change in the attitudes of a society leads to significant and important events that occur in the society. Shiller models the diffusion of opinion, or mood, through a population using a general epidemic model proposed by Nofsinger (2005) and Diener (2009). That is, the spreading of a mood is similar to the spread of a disease. The rate at which people are exposed to the changing attitudes and are persuaded is equal to the "infection rate." The parameters of the model can differ from one social movement to another causing some to be long lasting while others are short-lived. We argue that changes in social mood cause people to make different decisions. A change in mood may begin with some people undergoing a substantial change or by most people undergoing a small change. These people make decisions and act on this change in mood. Their interaction and communication with others causes further swings in mood in others. The collective decisions take time to appear in various ways. For example, a newfound optimism can be seen quickly in the stock market because it takes relatively little time and effort to trade. However, it takes longer for this optimism to be recorded in economic activity because of the additional time needed to plan and execute new business activity.

\subsection{Social mood on Weibo}

Each user submits periodic status updates, known as Weibo, that consist of short messages of maximum size 140 characters. These updates typically consist of personal information about the users, news or links to content such as images, video and articles. Every day, there are thousands of hundreds of Weibo updates on web, which will become a huge collective big data.

Mood word lists are one of the tools frequently used in sentiment analysis to detect a mood or classify emotions within a text. Such lists contain a set of words with their emotional assessment which can be represented by a set of named tag, as shown in Table 1.

Table 1. Affective mood norms of words on Weibo from November 1, 2012 to December 1, 2013, those data are got by search engine google.

\begin{tabular}{|l|l|}
\hline English translation & Frequency of occurrence \\
\hline Happy & 78400 \\
\hline Good Mood & 9320 \\
\hline Boring & 36200 \\
\hline Angry & 36000 \\
\hline Bad Mood & 1040 \\
\hline
\end{tabular}

We use a dataset formed of messages from Weibo. Weibo contains a very large number of very short messages created by the users of this micro blogging platform. The contents of the messages vary from personal thoughts to public statements. Generally, we can split the Weibo messages into two sets in a simple way: "a positive set": formed of messages containing positive emotions, i.e. ':)'; “a negative set": formed of messages containing negative emotions, i.e. ':('.

Then, we count the occurrence of each word from the dataset and estimate influence based on the respective number of occurrences of the word in each set.

\section{PREDICTING THE ECONOMICS TRENDS BASED ON SOCIAL MOOD}

\subsection{Social mood model}

In our method, a predicting model based on social 
mood is proposed. Among thousands of hundreds of Weibo, that useful information created via microblog can be divided into two categories: the ones that directly mood relate to the stock and the other ones that relate to the generally emotions about daily life. Social mood is assumed to have valid influence on the SSE composite index only on the same day it is published. Using the five different moods as shown in table 1 , a social mood during a period can be got. In this paper, the model is used to predict the trends of the SSE composite index. We don't quantitative accurate calculation, and just qualitative analysis for determining the big trends of SSE composite index. This method is very different from those existed predicting stock algorithm. It is to present collective behavior got from social media to influence on the stock.

Those mood are directly related with term frequency (TF) and the inverse document frequency (IDF), so that terms occur more often in Weibo and/or rarer in other Weibo will be given a higher value. Moreover, those Weibo that only occur in one class but not in the other are given a higher value to help better distinguish between different classes. Examples of some the unique mood from Weibo directly related to stock that are considered as "positive economics trend" are respectively: establishment, accumulation, growth, etc; while the ones from "negative economics trend" are respectively: separate, discharge, impair, etc. Then the two groups are respectively trained and classified using SVM and their results feed into another SVM to produce the combined prediction of price trends.

\subsection{Results}

We first propose Social mood index to capture various aspects of public mood. Social mood index contains 5 elements, such as "Happy", "Good mood", "Boring", "Angry" and "Bad mood". These social mood indexes can be computed by TF and IDF. In a simple way, we use search engine, such as google or baidu, to achieve TF or IDF of the five mood words on Weibo every month from November 1, 2012 to December 1, 2013, as shown in figure 1.

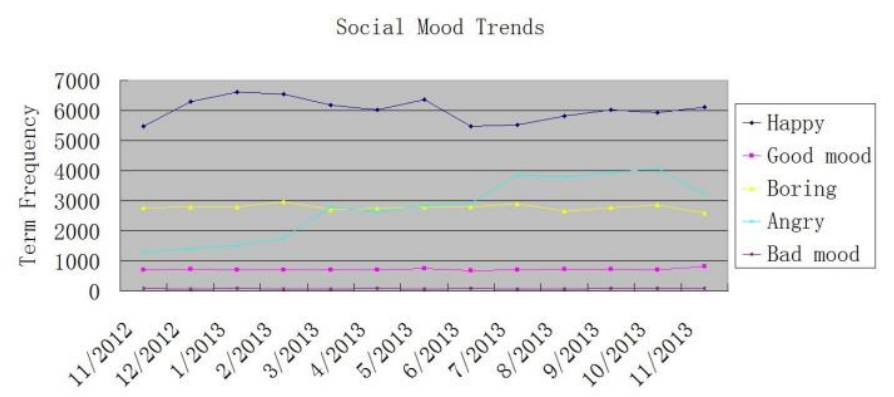

Figure 1. Tracking public mood states from Weibo posts to shows public response to $18^{\text {th }}$ National Congress of the Communist Party of China and $18^{\text {th }}$ National Congress of the Communist Party of China third plenary.
To visualize the correlation between social mood and the SSE composite index in more detail, we plot Fig. 2 (a). As can be seen in Fig. 2 both time series frequently overlap or point in the same direction. Changes in past values of "happy" mood can predict a similar rise or fall in SSE composite index. The "happy" mood dimension thus has predictive value with regards to the SSE composite index. In fact, i.e. May 30, 2013 to June 30 2013, is significantly drop area. On the other hand, December 3, 2012 to February 20, 2013, is clearly up area. The reason of up area can be used the social mood to analysis. During the period, $18^{\text {th }}$ National Congress of the Communist Party of China has been already convened. Thus, the "happy" mood and "good mood" are accounted for the most majority, as shown in figure 1. According to above behavior economics theory, the economics trend is transparently improved. Thus, the SSE composite index will increase. However, the drop area is because that "boring", "angry" and "bad mood" is accounted for the most majority, as shown in figure 2 . Finally, we can get much more information from those figures. Firstly, during November 1, 2012 to December 1, 2013, there are clearly twice fluctuations, which coincides the two important congress of the Communist Party of China. Secondly, Positive emotions are stronger than Negative emotions, which shows that people have strong confident about Chinese economy in future. Thirdly, "Happy" mood has a similar trend with the SSE composite index, which presents that "Happy" mood has the largest role in promoting economic. "Happy" mood doesn't only spread happiness but also can directly boost the economy. Finally, we found that social media indeed has a relationship with the economics, and we can use the relationship to forecast the economics trends in a relatively simple way.

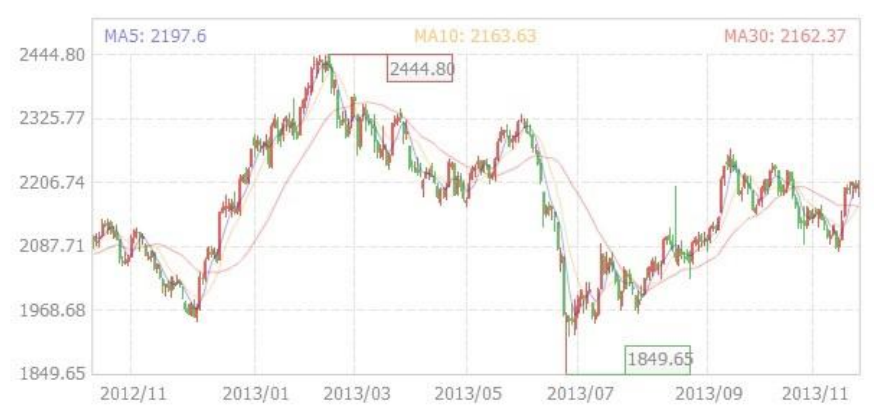

Figure 2. SSE composite index history data from netease (November 1, 2012 to December 1, 2013).

\subsection{Discussion}

In this paper, we investigate whether public mood as measured from large-scale collection of Weibo posted on weibo.com is correlated or even predictive of SSE composite index values. Our results show that changes in the public mood state can indeed be 
tracked from the content of large-scale Weibo feeds by means of rather simple text processing techniques and that such changes respond to a variety of sociocultural drivers in a highly differentiated manner. Among the 5 observed mood dimensions changes of the public mood along these mood dimensions match shifts in the SSE composite index values that occur a similar trends. We observe this effect of public mood states in terms of positive vs. negative mood. A Self-Organizing Fuzzy Neural Network can be used to train on the basis of past SSE composite index values and our public mood time series furthermore demonstrated the ability of the latter to significantly improve the accuracy of even the most basic models to predict SSE composite index values. Given the performance increase for a relatively basic model we are hopeful to find equal or better improvements for more sophisticated market models that may in fact include other information derived from different sources, and a variety of relevant economic indicators.

Public mood analysis from Weibo feeds on the other hand offers an automatic, fast, free and largescale addition that may in addition be optimized to measure a variety of dimensions of the public mood state. Our analysis does not acknowledge a number of important factors that will form the basis of future research. Furthermore, our method only predicts for the big trends of the SSE composite index, which cannot give much more detail about the trends. In future research, we will focus on quantitative analysis based on an improved method.

However, our method is a simple way to determine the economic trends, which can give accurate results about the increase values or decrease values. In this way, our method is only an additional method to existed institute market mechanisms. But, social computing is on its rising phase, which means a new future for human society. We firmly believe social media can have a much more important effect on understanding ourselves economic activities, which can help us to develop or adjust economics in a smart way.

\section{CONCLUSION}

In this paper, Weibo releases are combined with the technical indicators to predict daily direction of a stock price using social mood. The case study results showed that both the prediction performance and the profitability of the system are enhanced. However, the current system only categorizes the output into simple rise/fall without specifying the level of change. Therefore, future research efforts will focus on refining the prediction of price trends. Furthermore, the general applicability of the system also needs to be examined further by applying it to other stocks in different sectors.

\section{ACKNOWLEDGEMENT}

This work was supported by a grant from the Research Foundation of Education Bureau of Hubei Province China (Q20141507), a grant from the Natural Science Foundation of Hubei Province of China (2014CFB771), China Postdoctoral Science Foundation (2014M562017), and Research Project of Wuhan Institute of Technology, College of Post and Telecommunication (201403).

\section{REFERENCES}

[1] Bollen J, Mao H, Zeng X. Twitter mood predicts the stock market. Journal of Computational Science, 2011, 2(1): 18.

[2] Boyacioglu M A, Avci D. An adaptive network-based fuzzy inference system (ANFIS) for the prediction of stock market return: the case of the Istanbul stock exchange. Expert Systems with Applications, 2010, 37(12): 7908-7912.

[3] Vui C S, Soon G K, On C K, et al. A review of stock market prediction with artificial neural network (ANN). Control System, Computing and Engineering (ICCSCE), 2013 IEEE International Conference on. IEEE, 2013: 477-482.

[4] Garcia D. Sentiment during recessions. The Journal of Finance, 2013, 68(3): 1267-1300.

[5] Van Kleef G A, De Dreu C K W, Manstead A S R. An interpersonal approach to emotion in social decision making: The emotions as social information model. Advances in experimental social psychology, 2010, 42: 45-96.

[6] Fama E F. Market efficiency, long-term returns, and behavioral finance. Journal of financial economics, 1998, 49(3): 283-306.

[7] Soroka S N. Good news and bad news: Asymmetric responses to economic information. Journal of Politics, 2006, 68(2): 372-385.

[8] Nofsinger J R. Social mood and financial economics. The Journal of Behavioral Finance, 2005, 6(3): 144-160.

[9] Diener E, Ryan K. Subjective well-being: a general overview. South African Journal of Psychology, 2009, 39(4): 391-406. 